\title{
Probiotic Bifidobacterium lactis Probio-M8 treated and prevented acute RTI, reduced antibiotic use and hospital stay in hospitalized young children: a randomized, double-blind, placebo-controlled study
}

\author{
Mageswaran Uma Mageswary ${ }^{1} \cdot$ Xin-Yee Ang ${ }^{1} \cdot$ Boon-Kiat Lee ${ }^{1}$-Yi-Li Fiona Chung ${ }^{1}$ - Siti Nur Afiqah Azhar ${ }^{2}$. \\ Intan Juliana Abd Hamid ${ }^{3}$. Hafizi Abu Bakar ${ }^{1}$. Nurhanis Syazni Roslan ${ }^{2}$. Xiaojun Liu ${ }^{6}$. Xiaohong Kang ${ }^{7}$. Lu Dai ${ }^{7}$. \\ Sasidharan Sreenivasan ${ }^{8} \cdot$ Fahisham Taib $^{2,5} \cdot$ Heping Zhang ${ }^{4} \cdot$ Min-Tze Liong ${ }^{1}$ (I)
}

Received: 9 July 2021 / Accepted: 28 September 2021 / Published online: 26 November 2021

(c) The Author(s), under exclusive licence to Springer-Verlag GmbH Germany 2021

\begin{abstract}
Purpose The development of probiotics has seen tremendous growth over the years, with health benefits ranging from gut health to respiratory. We thus aimed to investigate the effects of probiotic Bifidobacterium lactis Probio-M8 $\left(2 \times 10^{10} \mathrm{log}\right.$ CFU/day) against acute respiratory tract infections (RTI), use of antibiotics, hospitalization period and elucidate the possible mechanisms of action in hospitalized young children.

Method A prospective, randomized, double-blind and placebo-controlled study was performed in RTI-hospitalized children. Patients were randomized to either the probiotic ( $n=60$, mean age $13.81 \pm 0.90$ months) or placebo $(n=60$, mean age $12.11 \pm 0.73$ months) which were administered upon admission, continued during hospitalization and 4-week post-discharged. RTI and gut health parameters were assessed at these time points using validated questionnaires while concentrations of inflammatory cytokines were assessed via oral swabs.

Results Probio-M8 reduced the duration of nasal, pharyngeal and general flu-like symptoms compared to the placebo during the hospitalization period and 4-week post-discharged $(P<0.05)$ as compared to the placebo, with a more prevalent effect against lower respiratory tract infections (LRTI). Probio-M8 reduced prescription of antibiotic $(P=0.037)$, prevented new prescription of antibiotic in non-prescribed patients $(P=0.024)$ and reduced hospitalization period in antibiotic-prescribed patients $(P=0.004)$ as compared to the placebo. Oral cytokine levels of TNF- $\alpha$ decreased in the Probio-M8 group $(P=0.001)$ accompanied by increased in IL-10 $(P=0.018)$ over 4-week post-discharged, while the placebo group did not exhibit such an effect. Increased IL-10 in the Probio-M8 group was correlated with decreased body ache $(r=-0.296, P=0.001)$, headache $(r=-0.295, P=0.001)$ and pain during swallow $(r=-0.235, P=0.010)$.

Conclusion Data from our present study show that $B$. lactis Probio-M8 could be a potential natural and non-drug strategy for the management of RTI in young children in a safe manner.

Clinical trial registration Clinical studies (Approval No. USM/JEPeM/19030177) were registered at ClinicalTrials.gov (Identifier No. NCT04122495) on September 30, 2019.
\end{abstract}

Keywords Bifidobacterium lactis Probio-M8 · Children · Respiratory tract infections · Probiotic · Inflammation · Antibiotic $\cdot$ Hospitalization

Fahisham Taib

fahisham@usm.my

$\triangle$ Heping Zhang

hepingdd@vip.sina.com

Min-Tze Liong

mintze.liong@usm.my

Extended author information available on the last page of the article

\section{Introduction}

Probiotics are live microorganisms that grant health effects to the host upon consumption in sufficient amounts [1]. Probiotic bacteria, which beneficially affect the host by improving the intestinal microbial balance, may affect the immune response; thus, boosting the body system to combat against diseases [2]. 
Respiratory tract infection (RTI) is an infectious disease that includes both the upper and lower respiratory tract. Upper respiratory tract infection (URTI) is less severe than lower respiratory tract infection (LRTI); however, the latter often requires hospital admissions. RTIs remain the top three causes of death and disability among infants and children globally. LRTI causes nearly four million deaths annually among children under 5 years old and may predispose for chronic respiratory diseases later in life [3]. Considering that acute RTIs are one of the leading causes of hospitalization and emergency department visits, the use of antibiotics in RTIs remains one of the main concerns in clinical practises. Antibiotic overuse is associated with an increase in the prevalence of antibiotic resistance, where 25,000 patients in Europe die annually following infections related to antibiotic-resistant microorganisms. Antibiotics were prescribed in $37 \%$ of children in The Netherlands and Israel [4]. Hospital-acquired infections have also been a major concern, especially among children who require prolonged hospitalization due to their susceptibility to increased risks of multidrug-resistant infections and overuse of antibiotics.

It is hoped that natural dietary interventions could buffer and reduce the dependency towards antibiotics in RTI and thus reducing hospitalization period, especially in young children. Children receiving either probiotic in milk or control milk showed reduced absentia from day care attributed to fewer RTI complications and less antibiotic requirement [5]. The duration of respiratory symptoms was significantly shorter in the probiotic group compared with the control group [2].

The precise mechanisms of action of probiotics in the management of RTI remain largely unknown. It is hypothesized that probiotics prevented inflammation in the epithelium of respiratory barriers; thus, preserving functions, cellular immunity and activities of immune cells and macrophages in airways. Lactobacillus plantarum DR7 has been reported to alleviate symptoms of respiratory illnesses via the activation of natural killer cells [6] while Lactobacillus casei Zhang exerted mechanisms against respiratory illnesses via the activation of $\mathrm{T}$ cells and $\mathrm{B}$ cells [7]. In a long-term probiotic intervention period of 10 months in pre-school children attending day care, Bifidobacterium longum BB536 was shown to reduce the frequency of respiratory illnesses while reducing the duration of sore throat as compared to the placebo [8]. It is thereby suggested that probiotics may offer protection on the risk of early RTI and recurrences thereby may represent a simple, safe, effective, and affordable method for the preventing RTI in children [9].

Bifidobacterium is one of the best-known probiotic genera globally and is widely used in the dairy industries. Evidence from in vitro, in vivo, and clinical studies have supported the safety and health benefits of Bifidobacterium strains [10]. The ingestion of fortified yoghurt with B. longum was shown to reduce harmful bacteria such as Enterobacteriaceae, Streptococcus and C. perfringens, and alter the gut microbiota profile with a significant increase of beneficial microbes Bifidobacterium and Lactobacillus species [11] Such findings suggest that Bifidobacterium strains are safe with antimicrobial potentials against harmful pathogens, which may play a role in preventing and/or treating the onsets of RTI.

Bifidobacterium lactis Probio-M8 (Probio-M8) was isolated from human colostrum samples collected from 40 healthy lactating women in the Inner Mongolia. A total of 197 bacterial strains were isolated where the genera of Bifidobacterium accounted for $4.06 \%$ comprising of three species. Probio-M8 was selected based on excellent tolerance to gastric acid, intestinal fluid and bile salt of the digestive system [12]. We hypothesized that Probio-M8 may exert a certain level of protection against RTI in young children. Most reports on probiotics and RTI primarily emphasized on adults in general, while young children under the age of 5 years old remain highly susceptible to respiratory illnesses. This is crucial as recent developments on probiotics have revealed the importance of strain and host dependencies, and the impacts of these on the quality of life of the intended patients.

With proper management of RTI, probiotics offer protection on the risk of early and recurrences of RTI thereby represent a simple, safe, effective, and affordable method for respiratory health management in children [9]. Thus, in this study, we explored the effects of a Bifidobacterium probiotic in reducing duration and frequency of symptoms attributed to acute RTI, prescription of antibiotic, hospitalization period and inflammatory parameters in hospitalized children under two years old.

\section{Materials and methods}

\section{Bifidobacterium lactis Probio-M8 and placebo products}

Bifidobacterium lactis Probio-M8 and placebo products were manufactured under an ISO9001 certified manufacturing plant (JinHua YinHe Biological Technology Co. Ltd.,China). The product $B$. lactis Probio-M8 contains Bifidobacterium lactis Probio-M8 and maltodextrin as carrier while placebo contains only maltodextrin. Both probiotic Probio-M8 and placebo are identical in taste, appearance and appeared as light-yellow powder. Both Probio-M8 and placebo products were kept at storage temperature below $30{ }^{\circ} \mathrm{C}$ according to the recommendation of the manufacturer. 


\section{Selection of patients}

Patients were recruited from Hospital Universiti Sains Malaysia in Kubang Kerian, Malaysia. The inclusion criteria were all children aged 0-24 month who were hospitalized for acute RTI with either bacterial or viral-like RTI needing acute medical observation or management. Children who were on long-term medications of 6 months or more, or an underlying glucose-6-phosphate dehydrogenase deficiency, and uncommitted parents were excluded from the study. RTI diagnosis were made by the medical team and characterized as respiratory symptomatology of either upper or lower tract infections such as coryzal, fever, cough together with positive clinical examination findings which included bronchiolitis, croup, pneumonia, tonsillopharyngitis and laryngitis. Written informed consent was obtained from the parents prior to the start of the study.

\section{Study protocol}

This was a double-blind, randomised and placebo-controlled design study. Randomisation was conducted upon applying the inclusion and exclusion criteria. Qualified patients were randomised according to 1:1 ratio to the two arms of the study according to a computer-generated list, assigned to the probiotic group and placebo group with treatment codes. Randomisation was performed by the study statistician, who had no contact with the participants. The allocation sequence was not available to any member of the research team until the completion of the study. Intervention consisted of daily administration of one sachet/day of Probio-M8 or placebo, starting from during admission at hospital and continued for 4 weeks upon discharged. Each sachet was two grams in total weight and contained $2 \times 10^{10} \log$ CFU of Probio-M8. Probio-M8 or placebo powder was dissolved in a small quantity of room temperature water or milk prior to consumption.

The primary outcome was duration of RTI symptoms, while secondary outcomes included parameters of gastrointestinal, RTI symptoms and concentrations of inflammatory proteins from the oral swabs. Compliance was monitored by requesting the participated parents to return all sachets, opened and unopened during the 4-week follow-up postdischarged. This study was conducted according to the guidelines laid down in the Declaration of Helsinki. All procedures involving human subjects were approved by the JEPeM-USM Review Panel on Clinical Studies (Approval No. USM/JEPeM/19030177) and was registered at ClinicalTrials.gov (identifier No. NCT04122495).

The sample size was calculated for a parallel group study design involving one prevention arm and one placebo arm and was based on power design analysis. A total of 120 subjects are needed for this study, with a total of 60 subjects in each arm and an additional 10\% dropout. This calculation was based on the need for a continuous response variable from an independent control and experimental subjects, with a ratio of control to subject fixed at $1: 1$, probability of 0.95 and Type-I error probability associated with this test of null hypothesis of 0.05. Previous data have shown that for an intervention using probiotic against reducing clinical visits in children for respiratory diseases, a standard deviation of 0.46 times within group was observed, accompanied by a reduction of 0.32 times between treatment and placebo groups [13].

\section{Analyses}

\section{Questionnaires}

Enrolled patients were assessed using three types of questionnaires; (i) demographic questionnaire which was collected upon admission (baseline), (ii) respiratory health questionnaire [14] which was collected at baseline, prior to discharged and on the next visit at 4-week post-discharged, and (iii) as probiotic products primarily enhance gut health, a gut health questionnaire [15] which was collected at similar time points. The questionnaires were multiscale where the respiratory health questionnaire comprised of two domains covering aspects on daily diet (5 items), clinical respiratory parameters (21 items), while the gut health questionnaire covered aspects of clinical gastrointestinal parameters (15 items). The domains for gastrointestinal and RTI questionnaire were identified through literature reviews, focus groups and opinions of experts, prior to generating the items for each tool. The translated Malay versions showed item content validity index (I-CVI) of 1.00 and 0.99 , respectively, and itemlevel face validity index (I-FVI) of 1.00 for both questionnaires, showing reliability and suitability for assessing gastrointestinal and RTI symptoms in young children [16]. Length of hospitalization period was obtained from hospital records.

\section{Inflammatory cytokines of oral samples}

Oral swab was collected from each child at three different time points; at baseline (during admission), at the time of discharge and at week-4 post-discharged, from the inside of both left and right cheeks, one immediately after the other. This was performed by our medical team and swabs were immediately placed in sterile saline and frozen at $-80{ }^{\circ} \mathrm{C}$ until further analyses. Oral swab samples were analyzed for the concentrations of interleukin-4 
and -10 , tumor necrosis factor (TNF)- $\alpha$ and interferon (IFN)- $\gamma$ using enzyme-linked immunosorbent assay (ELISA) kits (Immunodiagnostik, Germany) following the manufacturer's instructions. Changes in concentrations were determined by comparing the concentrations at baseline versus at the time of discharge or week-4 post-discharged.

\section{Statistical analyses}

Data were analyzed using SPSS version 24.0 (SPSS Inc., Chicago, IL). The primary hypothesis of this study involved differential efficacy between the two treatment groups of probiotic and placebo. Considering the skewed distribution and non-parametric nature of our data, differences of scale data between probiotic and placebo groups or between two different time points, were compared using the Mann-Whitney $U$ test, while nominal data were compared using the Chi-square test. Spearman's rank correlation was used in non-parametric correlation analyses with rho $(r)$ as the correlation coefficient. All tests were two-sided with $P<0.05$ as considered statistically significant and data are presented as mean value \pm standard error unless stated otherwise.

\section{Results}

\section{Baseline characteristics}

During the study period, 137 patients were hospitalized with various RTI diagnosis. A total of 17 patients dropped out during the 4-week period, primarily due to travel restrictions attributed to lockdown for COVID-19; thus, unable to perform sampling and collection. This has yielded 120 subjects after the 4-week study (Fig. 1; Probio-M8, $n=60$; placebo, $n=60)$. No adverse effects or any health implications complaints were received, indicating product safety. Although sufficient sample size was achieved; this sample size was calculated based on data of clinical visits. However, due to travel restrictions during lockdown for COVID-19, the assessment on clinical visits were unable to be performed. Insignificant differences were observed in all the general characteristics of Probio-M8 and placebo subjects (Table 1).

\section{RTI incidences and symptoms duration}

Probio-M8 reduced several RTI symptoms in all children during the warded period, with a more prevalent effect on pharyngeal symptoms (Table 2), where all pharyngeal
Fig. 1 Consort flowchart detailing patients' recruitment, randomization and allocation

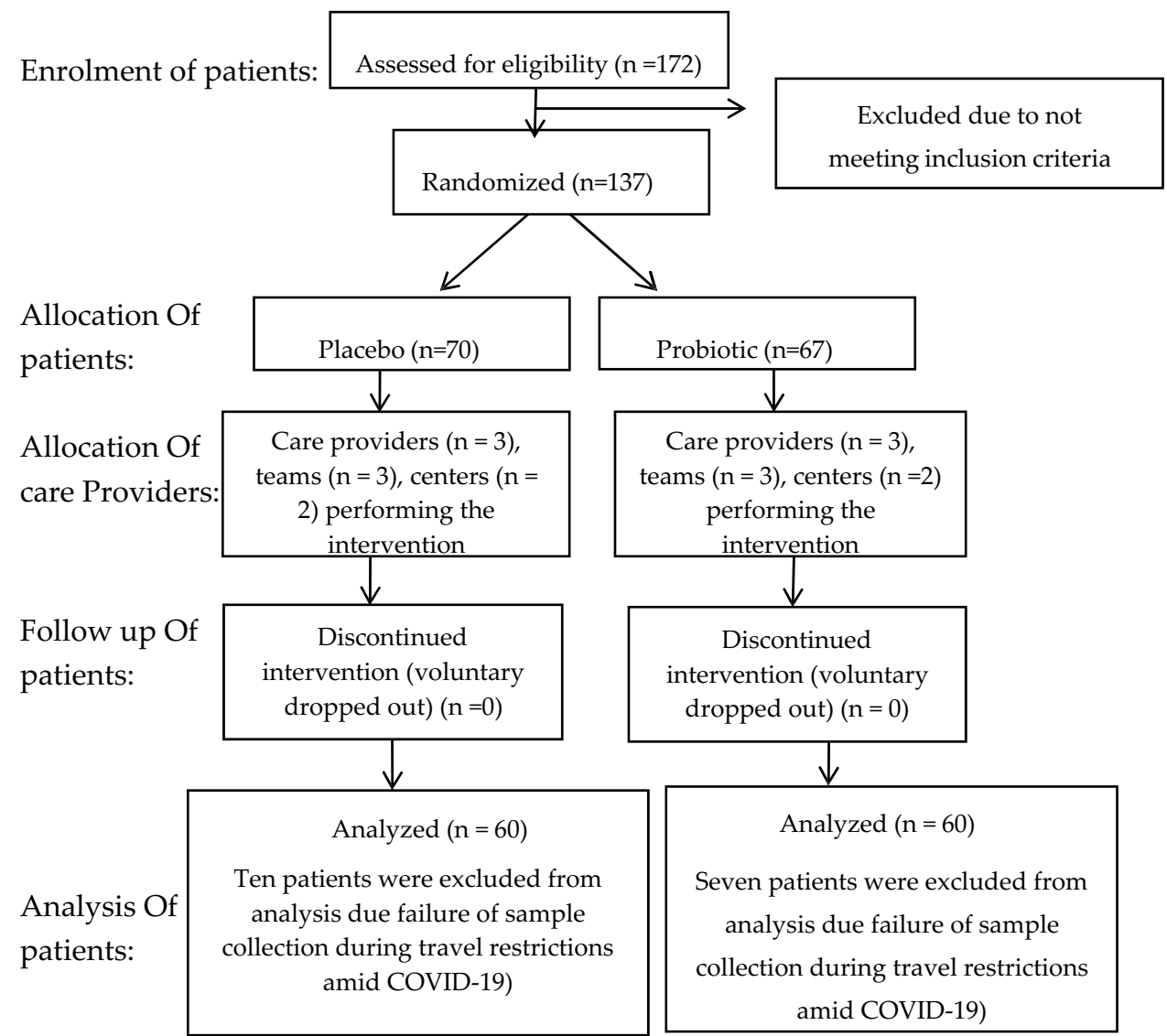


Table 1 Baseline characteristics of children $(n=120)$ hospitalized for acute RTI and randomly assigned to a double-blind treatment with either placebo $(n=60)$ or Bifidobacterium lactis Probio-M8 $(n=60)$

\begin{tabular}{|c|c|c|c|}
\hline Baseline characteristics & Placebo & $\begin{array}{l}\text { Probiotic Bifidobacterium lactis } \\
\text { Probio-M8 }\end{array}$ & $P$ value* \\
\hline All subjects $(n)$ & 60 & 60 & $0.000 * *$ \\
\hline LRTI $(n)$ & 50 & 42 & $0.084 * *$ \\
\hline URTI $(n)$ & 10 & 18 & $0.084 * *$ \\
\hline Sex(male) & $65 \%$ & $65 \%$ & $0.000 * *$ \\
\hline Age (months) & $12.11 \pm 0.73$ & $13.81 \pm 0.90$ & 0.262 \\
\hline Body weight (kg) & $10.70 \pm 1.40$ & $8.79 \pm 0.26$ & 0.781 \\
\hline Height (cm) & $72.18 \pm 1.55$ & $73.02 \pm 1.68$ & 0.384 \\
\hline Number of siblings & $2.93 \pm 0.21$ & $2.85 \pm 0.18$ & 0.922 \\
\hline Smokers in family & $0.53 \pm 0.06$ & $0.60 \pm 0.06$ & 0.460 \\
\hline History of food allergy & $0.07 \pm 0.03$ & $0.1 \pm 0.04$ & 0.514 \\
\hline Hospitalization for the past 12 months & $0.37 \pm 0.06$ & $0.30 \pm 0.06$ & 0.443 \\
\hline Incidence of diarrhea for the past 12 months & $0.33 \pm 0.06$ & $0.28 \pm 0.06$ & 0.562 \\
\hline Incidence of RTI for the past 12 months & $0.57 \pm 0.06$ & $0.48 \pm 0.07$ & 0.361 \\
\hline \multirow[t]{2}{*}{ Having pets at home } & $0.43 \pm 0.06$ & $0.37 \pm 0.06$ & 0.460 \\
\hline & $\%(n)$ & $\%(n)$ & $P$ value** \\
\hline \multicolumn{4}{|l|}{ Family income status: } \\
\hline Low $(<$ RM 2300) & $68.33(41)$ & $55.93(34)$ & 0.190 \\
\hline Middle (RM 2301-5999) & $26.67(16)$ & $30.51(18)$ & 0.641 \\
\hline$>$ RM6,000 & $3.33(2)$ & $11.86(7)$ & 0.082 \\
\hline$>$ RM10,000 & $1.67(1)$ & $1.69(1)$ & 0.991 \\
\hline \multicolumn{4}{|l|}{ Number of people living together in a household } \\
\hline Less than or equal to 4 people & $36.67(22)$ & $30.51(18)$ & 0.441 \\
\hline More than 4 people & $63.33(38)$ & $69.49(42)$ & 0.441 \\
\hline \multicolumn{4}{|l|}{ House location: } \\
\hline Urban & $35(21)$ & $32.20(19)$ & 0.701 \\
\hline Sub-urban & $40(24)$ & $40.68(25)$ & 0.850 \\
\hline Town & $25(15)$ & $27.12(16)$ & 0.832 \\
\hline
\end{tabular}

* $P$ value obtained via Mann-Whitney $U$ test unless specified otherwise

** $P$ value obtained via Chi-square test

symptoms studied have shown reduction in durations $(P<0.05)$ as compared to the placebo. Probio-M8 also reduced the duration of several nasal symptoms such as sneezing $(P=0.017)$ and runny nose $(P<0.0001)$ while the placebo group did not show any changes. A marginal reduction was also observed for nose block $(P=0.051)$ and mucus production $(P=0.059)$ in patients consuming Probio-M8 as compared to the placebo group. The duration of general flulike symptoms such as body ache and headache were also reduced in the Probio-M8 group as compared to the placebo counterpart $(P<0.05)$, with a marginal reduction in duration of vomit $(P=0.080)$.

At 4-week post-discharged, the administration of Probio-M8 has shown reduction in the duration of pharyngeal symptoms such as sore throat $(P=0.004)$ and pain during swallowing $(P=0.008)$ while the placebo group did not show such a benefit. Duration of general flu symptoms such as body ache $(P=0.012)$ and headache $(P=0.012)$ were also reduced in the Probio-M8 group as compared to the placebo counterpart.

Such effects of Probio-M8 were more prevalently observed in patients with LRTI (Table 3) than URTI (Table 4) during the hospitalization period. Probio-M8 reduced duration for runny nose, wheezing, sore throat, hoarseness, cough and fatigue in LRTI patients as compared to placebo $(P<0.05)$. Significant effects were not observed at 4-week post-discharged for LRTI patients between treatment groups. In patients with URTI, ProbioM8 reduced the number of RTI incidences, and duration for poor appetite and fatigue as compared to the placebo group during the hospitalization period $(P<0.05)$. ProbioM8 showed better benefits in patients with URTI at 4-week post-discharged, where duration of nose block, wheezing, 


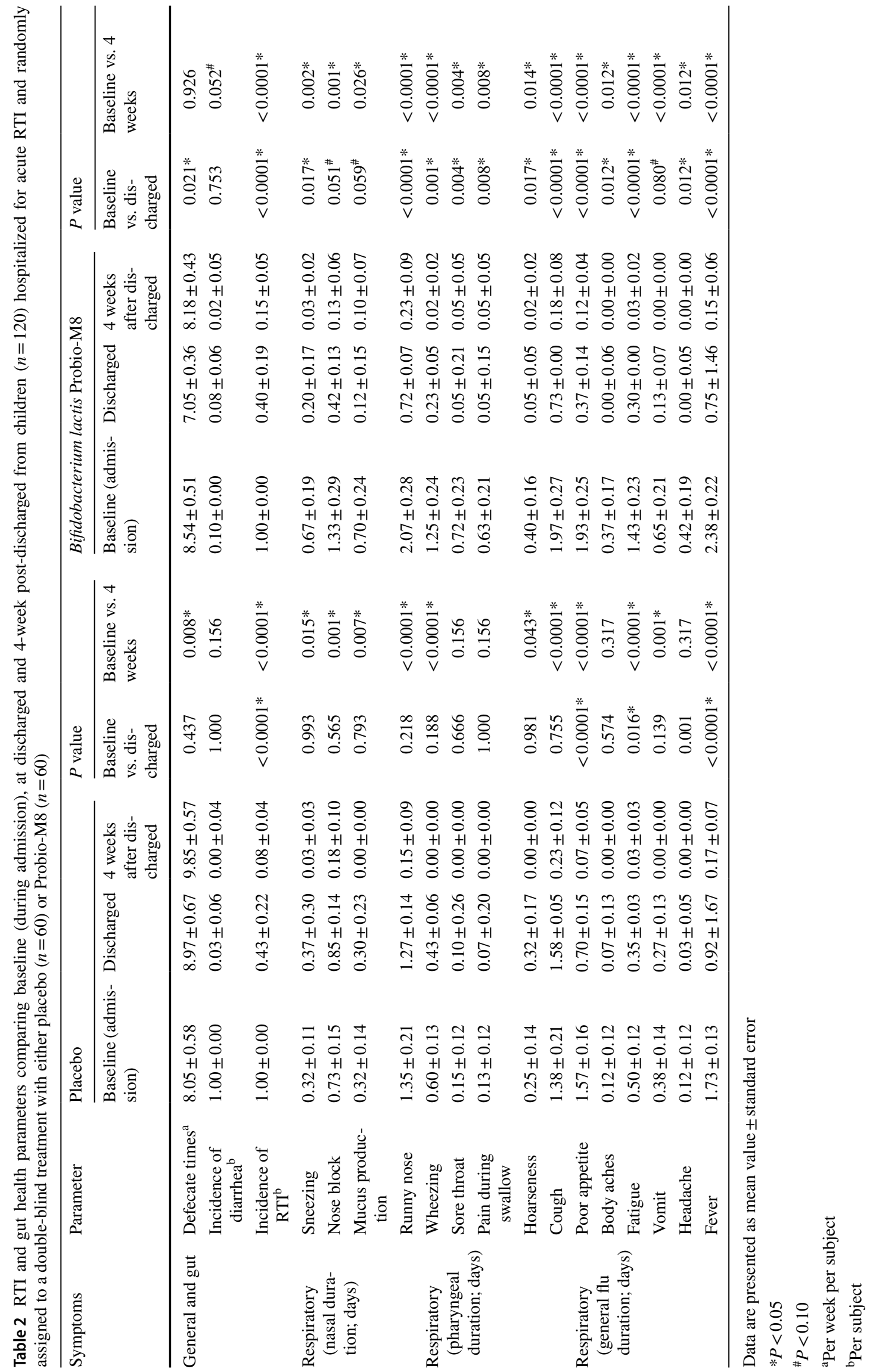


政

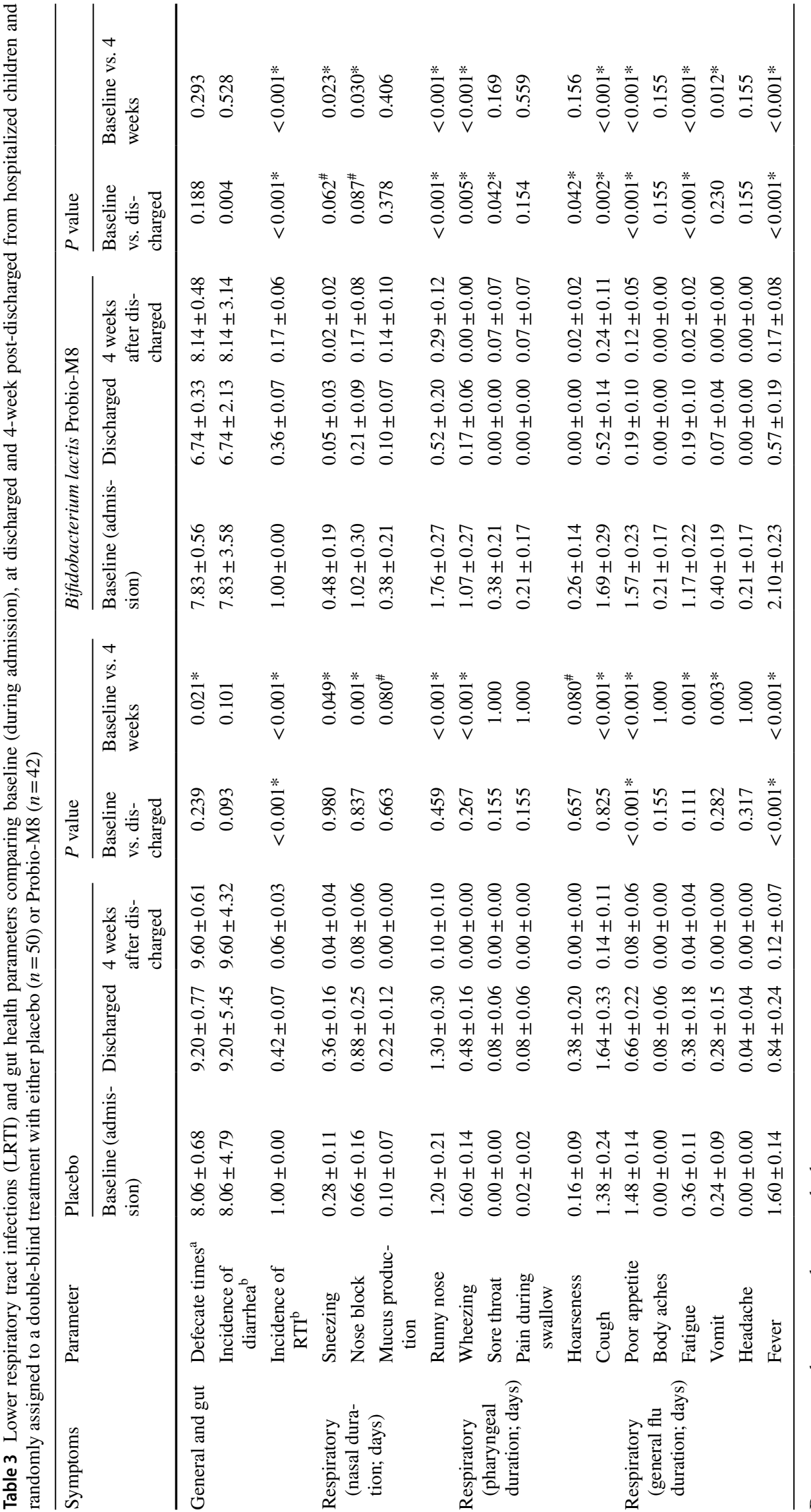

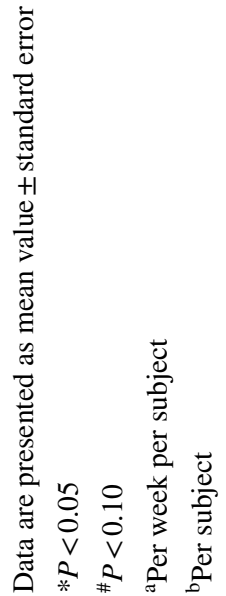


政

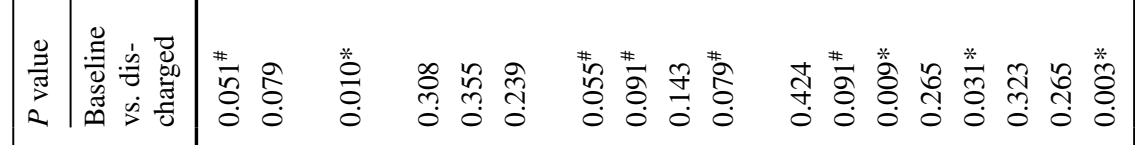
|

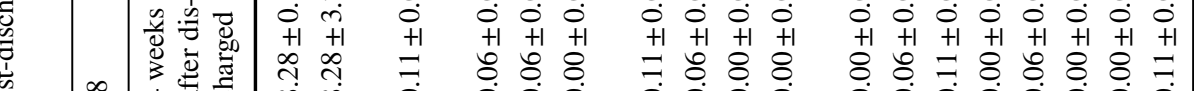

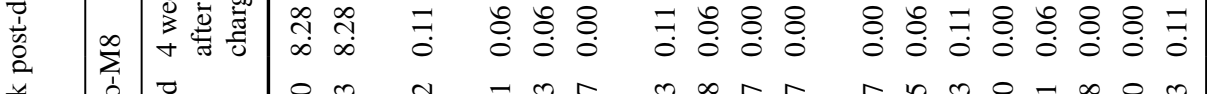

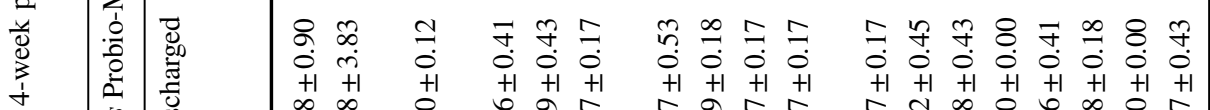

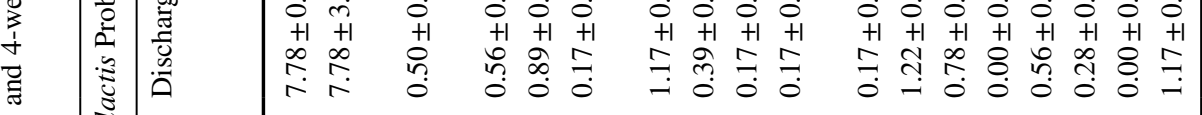
至 敢

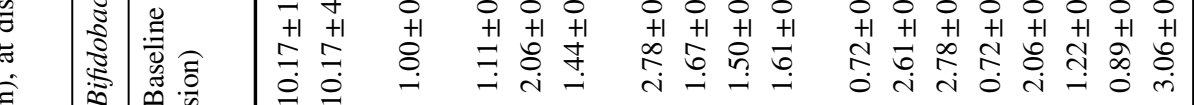

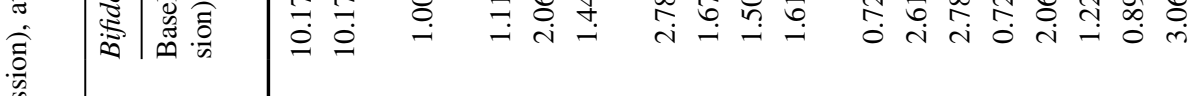

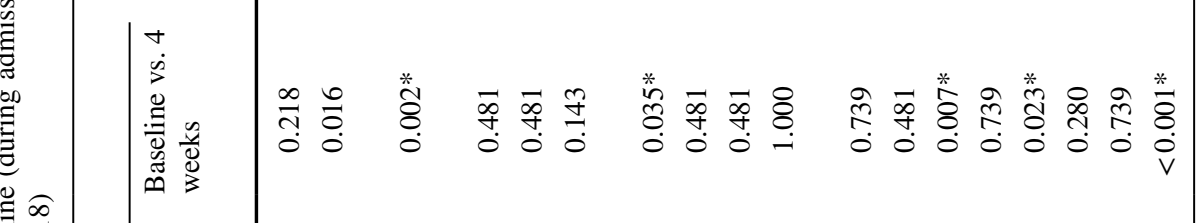
$\stackrel{0}{\infty}$

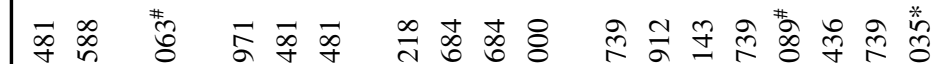

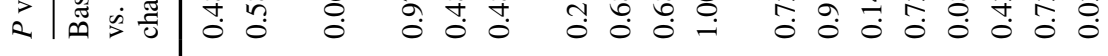

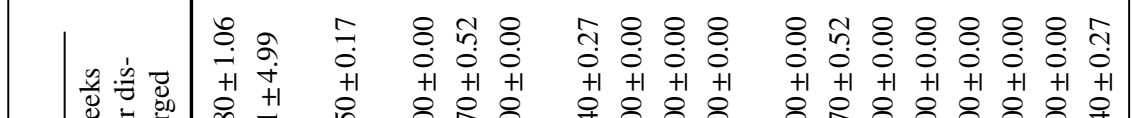

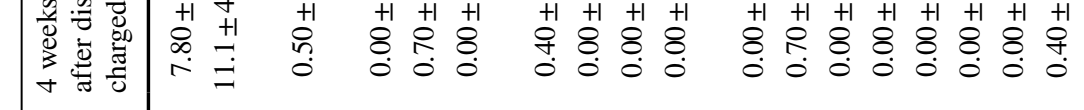

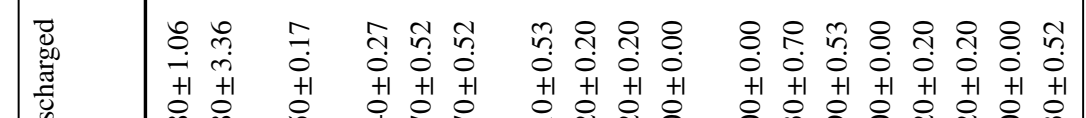

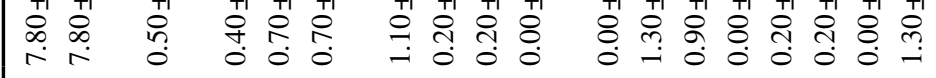
它

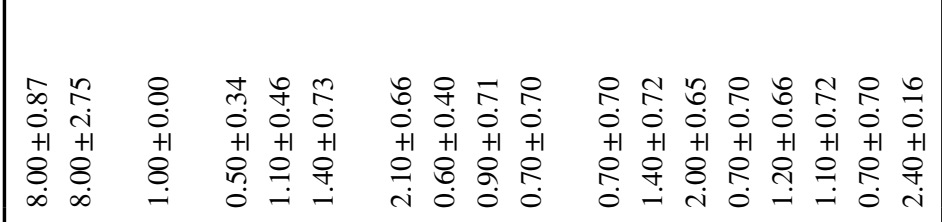
苛

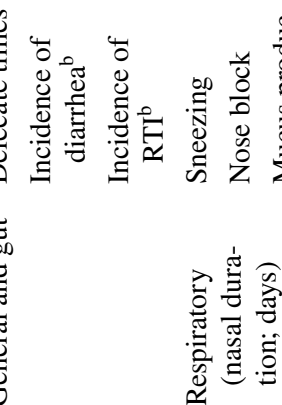

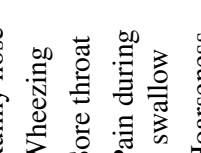

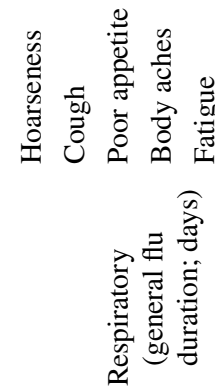

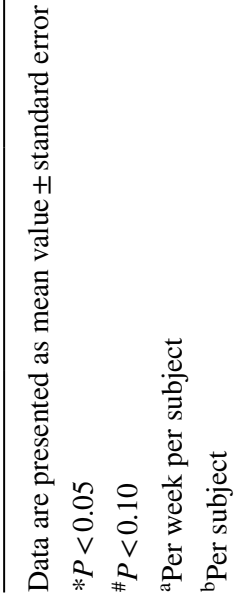


pain during swallow, cough and vomit were reduced as compared to the placebo $(P<0.05)$.

\section{Prescription of antibiotic and duration of hospitalization}

A total of $21.67 \%$ patients from placebo and $20.00 \%$ from Probio-M8 group were prescribed with antibiotic during admission (Table 5). Probio-M8 reduced the overall number of patients with prescribed antibiotic upon discharged $(P=0.037)$, with a more prevalent effect in LRTI patients $(P=0.001)$ as compared to the placebo. Although the Probio-M8 group had a higher number of URTI patients with prescription of antibiotic during admission, the number maintained at discharged while the placebo group showed a marginal increase $(P=0.088)$. The number of overall, LRTI and URTI subjects at 4 -week post-discharged was similar between treatment groups. A total of $25.00 \%$ of the patients who were previously prescribed with antibiotic has been stopped on the prescription at the time of discharge in the Probio-M8 group while all patients in the placebo group had to continue antibiotic treatment at the time of discharge $(P<0.0001)$. At 4 -week post-discharged, more patients previously started on antibiotic did not continue prescription in the Probio-M8 group (83.33\%) than the placebo counterpart $(69.23 \% ; P=0.021)$. Meanwhile, total number of subjects that were not initially prescribed with antibiotic during admission increased by $6.38 \%$ at discharge in the placebo group while Probio-M8 prevented new antibiotic prescription at discharge $(P=0.013)$. Such an effect was not observed at 4-week post-discharged. Among all patients prescribed with antibiotic during admission, all patients were hospitalized for a duration of 1 week or less in the ProbioM8 group while $7.69 \%$ of the placebo group were hospitalized for over 7 days $(P=0.004)$. Such an effect was not observed for hospitalization duration of 2 days or less.

\section{Inflammatory cytokines of oral samples}

Oral swab samples were evaluated for concentrations of cytokines, which included pro-inflammatory cytokines such as tumour necrosis factor- $\alpha(\mathrm{TNF}-\alpha)$ and interferongamma (IFN- $\gamma$ ), and anti-inflammatory cytokines such as interleukin-4 (IL-4) and interleukin-10 (IL-10). One sample from the probiotic group had concentrations of proteins that were below detection threshold and thus, only 59 samples from the probiotic group were included in the analyses. Patients consuming Probio-M8 showed a lesser increase in concentration of TNF- $\alpha$ from oral samples at 4-week post-discharged as compared to a higher increase as observed in the placebo group ( $P=0.001$; Fig. 2$)$. This was also accompanied by a higher increase in concentration of IL-10 from oral samples of subjects from the Probio-M8 group at 4-week post-discharged as compared to the placebo group $(P=0.018)$. Meanwhile, changes in concentrations of IFN- $\gamma$ and IL-4 from oral samples were insignificant between groups after 4 weeks. No significant changes were

Table 5 Prescription of antibiotic and effects on hospitalization period from hospitalized children whom were randomly assigned to a doubleblind treatment with either placebo or Bifidobacterium lactis Probio-M8

\begin{tabular}{|c|c|c|c|c|c|c|c|c|c|}
\hline \multirow[t]{2}{*}{ Prescription of antibiotic } & $\underline{\text { Placebo }}$ & Probio-M8 & \multirow[t]{2}{*}{$P$ value } & Placebo & Probio-M8 & \multirow[t]{2}{*}{$P$ value } & Placebo & Probio-M8 & \multirow[t]{2}{*}{$P$ value } \\
\hline & \multicolumn{2}{|c|}{ Admission/baseline } & & \multicolumn{2}{|c|}{ Discharged } & & \multicolumn{2}{|c|}{$\begin{array}{l}4 \text { weeks after dis- } \\
\text { charged }\end{array}$} & \\
\hline Overall subjects prescribed with antibiotic & $21.67 \%$ & $20.00 \%$ & 0.728 & $26.67 \%$ & $15.00 \%$ & $0.037 *$ & $13.33 \%$ & $10.00 \%$ & 0.506 \\
\hline LRTI subjects prescribed with antibiotic & $21.67 \%$ & $13.33 \%$ & 0.094 & $25.00 \%$ & $8.33 \%$ & $0.001 *$ & $10.00 \%$ & $8.33 \%$ & 0.621 \\
\hline URTI subjects prescribed with antibiotic & $0.00 \%$ & $6.67 \%$ & $0.007 *$ & $1.67 \%$ & $6.67 \%$ & $0.088^{\#}$ & $3.33 \%$ & $1.67 \%$ & 0.651 \\
\hline $\begin{array}{l}\text { Subjects prescribed with antibiotic during } \\
\text { admission, and stopped prescription } \\
\text { thereafter }\end{array}$ & & & & $0.00 \%$ & $25.00 \%$ & $<0.0001 *$ & $69.23 \%$ & $83.33 \%$ & $0.021 *$ \\
\hline $\begin{array}{l}\text { New subjects prescribed with antibiotic, } \\
\text { but not prescribed during admission }\end{array}$ & & & & $6.38 \%$ & $0.00 \%$ & $0.013 *$ & $8.51 \%$ & $8.33 \%$ & 0.800 \\
\hline $\begin{array}{l}\text { Patients with antibiotic prescription at } \\
\text { admission }\end{array}$ & Placebo & Probio-M8 & $P$ value & & & & & & \\
\hline \multicolumn{10}{|l|}{ Hospitalization period (length of stay): } \\
\hline$\leq 2$ days & $69.23 \%$ & $58.33 \%$ & 0.106 & & & & & & \\
\hline$>2$ days & $30.77 \%$ & $41.67 \%$ & & & & & & & \\
\hline$\leq 7$ days & $92.31 \%$ & $100.00 \%$ & $0.004 *$ & & & & & & \\
\hline$>7$ days & $7.69 \%$ & $0.00 \%$ & & & & & & & \\
\hline
\end{tabular}




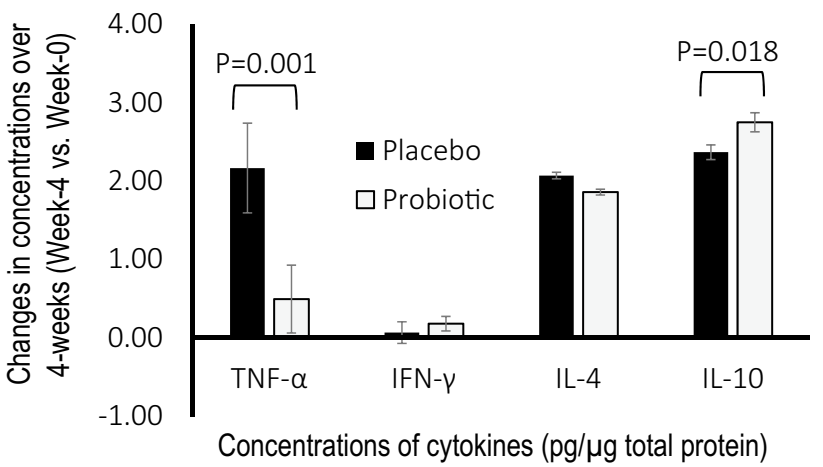

Fig. 2 Changes in concentrations of cytokines (pg/ $\mu \mathrm{g}$ total protein) such as tumor necrosis factor- $\alpha$ (TNF- $\alpha)$, interferon-gamma (IFN- $\gamma$ ), interleukin-4 (IL-4) and interleukin-10 (IL-10) in comparison of baseline and at 4-week post-discharged from oral swabs of children. Children were hospitalized for acute RTI and randomly assigned to a double-blind treatment with either placebo $(n=60)$ or probiotic Bifidobacterium lactis Probio-M8 $(n=59)$ upon admission to ward, throughout hospitalization and until 4-week post-discharged

observed between baseline and at discharged for both treatment groups (data not shown).

\section{Gut health parameters}

While the placebo group did not show changes in defecation frequency between baseline and at discharged, patients consuming Probio-M8 showed a reduction of defecation frequency $(P=0.021)$, that was not accounted for by constipation or diarrhea (Table 2). Insignificant changes were observed for incidences of diarrhea between treatment groups. The administration of Probio-M8 had maintained defecation frequency during the 4-week period upon discharged (between baseline and 4 weeks), while patients in the placebo group showed increased defecation frequency $(P=0.008)$. The administration of Probio-M8 also marginally reduced the incidences of diarrhea $(P=0.052)$ at 4-week post-discharged while the placebo group did not show such an effect. Meanwhile, in patients with LRTI (Table 3), the placebo group showed an increase in defecation frequency 4-week post-discharged $(P=0.021)$ while the Probio-M8 group showed insignificant changes. Insignificant changes were observed between groups in patients with URTI for both periods of discharged and 4-week post-discharged (Table 4).

\section{Correlation analysis}

Correlation analysis was performed for clinical symptoms against oral cytokines levels that yielded significant differences between treatment groups at 4-week post-discharged. Among the six significant clinical symptoms (defecation frequency, sore throat, body ache, vomit, headache, pain during swallow) and the two significant inflammatory cytokines (TNF- $\alpha$, IL-10), only clinical symptoms of body ache, headache and pain during swallowing against IL-10 showed significant correlations between treatment groups. The Probio-M8 group exhibited significant negative Spearman's correlation between body ache $(r=-0.296, P=0.001)$, headache $(r=-0.295, P=0.001)$ and pain during swallow $(r=-0.235, P=0.010)$ against IL-10, while no such correlations were observed for the placebo group $(P>0.05)$.

\section{Discussion}

RTIs in children should not be taken lightly, as their consequences often include parental absentia from work, increase in hospitalization and emergency room visits, antibiotic prescriptions and spread of pathogens and illnesses to other family members within the same household. The use of probiotics to treat and/or prevent RTI in children is not new, where probiotics are often seen as a feasible non-drug intervention to improve the immunity of children against RTI. However, it should be noted that not all probiotics have the same biological activity, but each has specific mechanism of action, efficacy, and more importantly, host-, dose- and strain-dependency. Various meta-analyses have shown contradicting results where some studies did not yield any significant benefits against respiratory illnesses in children [17].

In our present study, we aimed to establish the benefits of one specific strain of probiotic at a specific dose is administered to a specific group of patients. The observed beneficial effects within such designed specificity have enabled us to justify concerns related to host-, dose- and strain-dependency. The administration of Probio-M8 reduced the duration of the majority nasal, pharyngeal and general flu symptoms, as compared to the placebo during the hospitalization period. Meanwhile, several of these symptoms, primarily attributed to pain such as sore throat, pain during swallowing, body ache and headache have continuously improved in the Probio-M8 group over the next 4 weeks upon discharged as compared to the placebo counterpart, indicating potential prolonged benefits. LRTI often poses more life-threatening threats than URTI, with prolonged hospitalization period and the risk of starting on a broad spectrum of antibiotic use. Our present study showed that Probio-M8 reduced the duration of RTI symptoms more prevalently during the hospitalization period than at 4-week post-discharged, indicating that the severity of LRTI can be diminished in a speedy manner without prolonged detrimental effects in subsequent hospital visits.

The known causes of inflammation in the respiratory tract include immune activation in response to local infections, allergic responses to inhaled allergens, and trauma to the respiratory epithelium predominantly with the disruption 
of mucosal membrane integrity. Meanwhile, inflammation and infections in the respiratory tract were associated with an identifiable pathogen causes approximately one-third of RTI incidences [18]. The administration of Probio-M8 has benefited against pharyngeal irritation and odynophagia in young children, which were related to reduced inflammatory effects. Subjects in the placebo group showed higher concentration of oral pro-inflammatory cytokine TNF- $\alpha$ accompanied by lower concentration of plasma anti-inflammatory cytokine IL-10, indicating higher inflammation in the oral region consistent with sore throat and pain during swallowing, as compared to children who consumed Probio-M8 over 4-week period. Prolonged inflammation which may also include symptoms such as body ache and headache, with indications of an extended process in fighting against infections during the healing cycle. The observed oral cytokine profiles in our present study over 4 weeks; thus, exemplifying the importance of probiotics in reducing inflammation and subsequently duration of pain and respiratory symptoms, as also supported by the negative correlations of IL-10 with headache, body ache and pain during swallow. This is crucial as young children have been reported to feel pain like adults but have much lower pain thresholds [19], leading to much behavioural changes including tantrums and anxiety [20].

Considering that probiotics are orally consumed, much emphasis is given on gut health over the years. While probiotics have been well documented for gut health benefits such as general diarrhea, antibiotic-associated diarrhea, constipation and colitis, these effects were less prevalent in the present study. Notably, the administration of Probio-M8 has reduced defecation frequency in hospitalized children, while the placebo showed increased episodes during the hospitalization period. Considering that such a benefit did not account for improvement of diarrhea, we postulate that Probio-M8 may have prevented any possible increased in abnormal bowel movements during the hospitalization. It is not uncommon for children to experience diarrhea during hospitalization attributed to various factors such as antibiotics exposure, prolonged hospitalization, enteral feeding and the presence of antimicrobial stewardship programs in hospitals. Children are more likely to acquire infections upon hospitalization with over sixfold higher mortality rate than non-hospitalized children [21].

As antibiotic resistance is an increasing global concern, the prescription of antibiotic was thus evaluated in our present study. Of all patients that were prescribed with antibiotic in our present study, the administration of Probio-M8 evidently prevented further prescription upon discharge with a more prevalent effect in patients with LRTI. More importantly, with the administration of Probio-M8, we could see increased number of patients that did not require antibiotic prescription at discharge, indicating the potential protections against hospital acquired infections. While prolonged inpatient stay has been defined as greater than 1.8 days in paediatric setting [22, 23], acute LRTI and URTI patients often require a longer period of hospital stay. Children below 5 years old that were hospitalized for LRTI were shown to have a median hospitalization duration of 7 days [24], while children with mean age of 2.5 years old and hospitalized for acute URTI was shown to need 6.2 days of hospital stay [25]. In our present study, although the administration of probiotic had no effect in reducing hospital stay below 2 days in antibiotic-prescribed patients, Probio-M8 prevented prolonged hospitalization period of over 7 days, indicating a faster recovery and discharged than the placebo. Probiotics have a long history of safe use while the safety of vulnerable groups which include young children is always a primary concern. With no reported adverse effects or health complications in all subjects throughout the intervention period, we remain confident that the dose and probiotic strain selected are safe for the targeted group of young children below 2 years of age.

\section{Conclusions}

Our present study showed that the administration of Bifidobacterium lactis Probio-M8 $\left(2 \times 10^{10} \log\right.$ CFU/day $)$ daily during admission at hospital and continued 4-week postdischarged exhibited beneficial effects against acute RTI and gastrointestinal symptoms in children below 2 years old, both in terms of frequency and duration of symptoms as compared to the placebo. We postulate that these effects were mostly attributed to anti-inflammatory properties of Probio-M8 as shown by the modulation of oral cytokine levels and significant correlation analysis. More importantly, Probio-M8 reduced prescription of antibiotic, prevented new prescription of antibiotic in non-prescribed patients and reduced hospitalization period in antibiotic-prescribed patients. Taken altogether, our current study has illustrated dependency requirements of a probiotic strain namely strain-, host- and disease specific in a safe manner.

Acknowledgements We thank the director of Hospital Universiti Sains Malaysia (HUSM), Kubang Kerian, Kelantan for granting permission to use patients' medical records, space and assets belonging to the hospital for this project. We also thank Beijing Scitop Biotech Co., Ltd. for providing the probiotic and placebo products.

Author contributions M-TL and HZ conceived and designed the experiments. FT provided medical consultation and performed sampling. M-TL analyzed the data. MUM, X-YA, B-KL, Y-LFC, SNAA performed the study. MUM, HZ, FT and M-TL drafted the work and wrote the manuscript. IJAH, HAB, NSR, SS, XL, XK and LD revised critically for intellectual content. 
Funding Research Assistant scheme was supported by Universiti Sains Malaysia. Probiotic and placebo products were courtesy of Beijing Scitop Biotech Co., Ltd.

Data availability Data described in the manuscript, code book, and analytic code will be made available upon request pending approval by institutional review board. ClinicalTrials.gov (identifier number NCT04122495).

\section{Declarations}

Conflict of interest Xiaojun Liu is an employee of Beijing Scitop Biotech Co., Ltd.

Ethical statement This study was conducted according to the guidelines laid down in the Declaration of Helsinki. All procedures involving human subjects were approved by the JEPeM-USM Review Panel on Clinical Studies (Approval No. USM/JEPeM/19030177) and was registered at ClinicalTrials.gov (identifier No. NCT04122495).

Informed consent Written informed consent has been obtained from all subjects to publish this paper.

\section{References}

1. Meeting JFWECoFA, Organization WH (2006) Safety evaluation of certain contaminants in food. Food \& Agriculture Org.; Available from: http://www.fao.org/3/at881e/at881e.pdf. Accessed 2 Aug 2021

2. Hatakka K, Savilahti E, Pönkä A, Meurman JH, Poussa T, Näse L, Korpela R (2001) Effect of long term consumption of probiotic milk on infections in children attending day care centres: double blind, randomised trial. BMJ 322(7298):1327. https://doi.org/10. 1136/bmj.322.7298.1327

3. Forum of International Respiratory Societies (2017) The global impact of respiratory disease-second edition. Sheffield, European respiratory society, (https://www.who.int/gard/publicatio ns/The_Global_Impact_of_Respiratory_Disease.pdf). Accessed 2 Aug 2021

4. van Houten CB, Cohen A, Engelhard D, Hays JP, Karlsson R, Moore E (2019) Antibiotic misuse in respiratory tract infections in children and adults - a prospective, multicentre study (TAILORED Treatment). Eur J Clin Microbiol Infect Dis 38(3):505514. https://www.ncbi.nlm.nih.gov/pmc/articles/PMC6394715/. Accessed 2 Aug 2021

5. Lehtoranta LM, Pitkäranta AN, Korpela R (2012) Probiotic Lactobacillus Rhamnosus GG and respiratory illness in children. Dietary Fibres \& Pre/Probiotics 23(6):4-7https://www.researchga te.net/profile/Liisa-Lehtoranta/publication/235550630_Probiotic_ Lactobacillus_rhamnosus_GG_and_respiratory_illness_in_child ren/links/09e41511ce946db46f000000/Probiotic-Lactobacillusrhamnosus-GG-and-respiratory-illness-in-children.pdf. Accessed 2 Aug 2021

6. Chong H-X, Yusoff NAA, Hor Y-Y, Lew L-C, Jaafar MH, Choi S-B (2019) Lactobacillus plantarum DR7 improved upper respiratory tract infections via enhancing immune and inflammatory parameters: a randomized, double-blind, placebo-controlled study. J Dairy Sci 102(6):4783-4797

7. Hor Y-Y, Lew L-C, Lau AS-Y, Ong J-S, Chuah L-O, Lee Y-Y (2018) Probiotic Lactobacillus casei Zhang (LCZ) alleviates respiratory, gastrointestinal \& RBC abnormality via immuno-modulatory, anti-inflammatory \& anti-oxidative actions. J Funct Foods. https://doi.org/10.1016/j.jff.2018.03.017

8. Lau AS-Y, Yanagisawa N, Hor Y-Y, Lew L-C, Ong J-S, Chuah L-O (2018) Bifidobacterium longum BB536 alleviated upper respiratory illnesses and modulated gut microbiota profiles in Malaysian pre-school children. Benef Microbes 9(1):61-70

9. Ahanchian H, Jones CM, Chen YS, Sly PD (2012) Respiratory viral infections in children with asthma: do they matter and can we prevent them? BMC paediatrics 12(1):1-12. https://doi.org/ 10.1186/1471-2431-12-147

10. Kondo J, Xiao JZ, Shirahata A, Baba M, Abe A, Ogawa K, Shimoda T (2013) Modulatory effects of Bifidobacterium longum BB536 on defecation in elderly patients receiving enteral feeding. WJG 19(14):2162. https://doi.org/10.3748/wjg.v19.i14.2162

11. Ogata T, Kingaku M, Yaeshima T, Teraguchi S, Fukuwatari Y, Ishibashi N, Iino H (1999) Effect of Bifidobacterium longum BB536 yogurt administration on the intestinal environment of healthy adults. Microb Ecol Health Dis 11:41-46. https://doi.org/ 10.1080/089106099435916

12. Liu W, Chen M, Duo L, Wang J, Guo S, Sun H, Zhang H (2020) Characterization of potentially probiotic lactic acid bacteria and bifidobacteria isolated from human colostrum. J Dairy Sci 103:4013-4025. https://doi.org/10.3168/jds.2019-17602

13. Weizman Z, Asli G, Alsheikh A (2005) Effect of a probiotic infant formula on infections in child care centers: comparison of two probiotic agents. Pediatrics 115(1):5-9. https://doi.org/10.1542/ peds.2004-1815

14. School of Industrial Technology, Universiti Sains Malaysia, Pulau Pinang, Malaysia, Amy Sie-Yik L, Yusoff MSB, School of Medical Sciences, Universiti Sains Malaysia, Kelantan, Malaysia, Yeong-Yeh L, School of Medical Sciences, Universiti Sains Malaysia, Kelantan, Malaysia (2017) Development, Translation and Validation of Questionnaires for Diarrhoea and Respiratoryrelated Illnesses during Probiotic Administration in Children. Educ Med J 19-30. http://eduimed.usm.my/EIMJ20170904/ EIMJ20170904_03.pdf. Accessed 2 Aug 2021

15. Liu G, Chong HX, Chung FYL, Li Y, Liong MT (2020) Lactobacillus plantarum DR7 modulated bowel movement and gut microbiota associated with dopamine and serotonin pathways in stressed adults. Int J Mol Sci 21(13):4608. https://doi.org/10.3390/ ijms21134608

16. Lau ASY, Yusoff MSB, Lee YY, Choi SB, Rashid F, Wahid N, Liong MT (2017) Development, translation and validation of questionnaires for diarrhea and respiratory-related illnesses during probiotic administration in children. Edu in Med J 9:19-30. https://doi.org/10.21315/eimj2017.9.4.3Esposito

17. Esposito S, Rigante D, Principi N (2014) Do children's upper respiratory tract infections benefit from probiotics? BMC Infect Dis 14(1):194. https://bmcinfectdis.biomedcentral.com/articles/ 10.1186/1471-2334-14-194. Accessed 2 Aug 2021

18. Gleeson M, Pyne DB (2016) Respiratory inflammation and infections in high-performance athletes. Immunol Cell Biol 94(2):124 131. https://doi.org/10.1038/icb.2015.100

19. Goksan S, Hartley C, Emery F, Cockrill N, Poorun R, Moultrie F, Slater R (2015) fMRI reveals neural activity overlap between adult and infant pain. Elife. https://doi.org/10.7554/elife.06356

20. Power NM, Howard RF, Wade AM, Franck LS (2012) Pain and behaviour changes in children following surgery. Arc Dis Child BMJ 97(10):879-884. https://doi.org/10.1136/archdischi ld-2011-301378

21. Khalil A, Hendaus MA, Elmagboul E, Mohamed A, Deshmukh A, Elmasoudi A (2019) Incidence of clostridium difficile infection and associated risk factors among hospitalized children in Qatar. Ther Clin Risk Manag 15:1343. https://doi.org/10.2147/TCRM. S229540 
22. Heys M, Rajan M, Blair M (2017) Length of paediatric inpatient stay, socio-economic status and hospital configuration: a retrospective cohort study. BMC Health Serv Res 17(1):274. https://doi.org/10.1186/s12913-017-2171-x (PMID: 28412944; PMCID: PMC5392919)

23. Sanchez-Codez MI, Moyer K, Benavente-Fernández I, Leber AL, Ramilo O, Mejias A (2021) Viral loads and disease severity in children with rhinovirus-associated illnesses. Viruses 13(2):295. https://doi.org/10.3390/v13020295
24. Tan KK, Dang DA, Kim KH, Kartasasmita C, Kim HM, Zhang XH, Shafi F, Yu TW, Meyer ELN (2018) Burden of hospitalized childhood community-acquired pneumonia: a retrospective crosssectional study in Vietnam, Malaysia, Indonesia and the Republic of Korea. Hum Vaccin Immunother 14(1):95-105

25. Sam IC, Abdul-Murad A, Karunakaran R, Rampal S, Chan YF, Nathan AM, Ariffin H (2010) Clinical features of Malaysian children hospitalized with community-acquired seasonal influenza. Int J Infect Dis 14:e36-e40

\section{Authors and Affiliations}

\section{Mageswaran Uma Mageswary ${ }^{1}$. Xin-Yee Ang ${ }^{1}$. Boon-Kiat Lee ${ }^{1}$ - Yi-Li Fiona Chung ${ }^{1}$. Siti Nur Afiqah Azhar ${ }^{2}$. Intan Juliana Abd Hamid ${ }^{3}$. Hafizi Abu Bakar ${ }^{1}$. Nurhanis Syazni Roslan ${ }^{2} \cdot$ Xiaojun Liu $^{6} \cdot$ Xiaohong Kang $^{7} \cdot$ Lu Dai $^{7}$. Sasidharan Sreenivasan ${ }^{8} \cdot$ Fahisham Taib $^{2,5} \cdot$ Heping Zhang ${ }^{4} \cdot$ Min-Tze Liong ${ }^{1}$ (1)}

1 School of Industrial Technology, Universiti Sains Malaysia, 11800 Penang, Malaysia

2 School of Medical Sciences, Universiti Sains Malaysia, 16150 Kelantan, Malaysia

3 Advanced Medical and Dental Institute, Universiti Sains Malaysia, 13200 Penang, Malaysia

4 Key Laboratory of Dairy Biotechnology and Engineering, Ministry of Education, Inner Mongolia Agricultural University, Hohhot 010018, Inner Mongolia, China
5 Hospital Universiti Sains Malaysia (HUSM), Health Campus, 16150 Kelantan, Malaysia

6 Beijing Scitop Biotech Co., Ltd., Beijing 101407, China

7 Inner Mongolia Mengniu Dairy (Group) Co. Ltd., Shengle Economic Zone, Helingeer, Huhhot 011500, Inner Mongolia, China

8 Institute for Research in Molecular Medicine (INFORMM), Universiti Sains Malaysia, 11800 Penang, Malaysia 